\title{
NOUVELLE
}

\section{Sévérité de l'hypertension hyperkaliémique familiale causée par les mutations de CUL-3}

\section{Une histoire de reins et de vaisseaux}

Chloé Rafael ${ }^{1,2}$, Juliette Hadchouel ${ }^{3,4}$
${ }^{1}$ Centre de recherche des Cordeliers, Inserm UMR-S1138, Sorbonne université, Université de Paris, 15 rue de l'École de Médecine, 75006 Paris, France.

${ }^{2}$ CNRS ERL 8228 - Laboratoire de physiologie rénale et tubulopathies, 75006, Paris, France.

${ }^{3}$ Inserm UMR_S1155, Hôpital Tenon, 4 rue de la Chine, 75020 Paris, France.

${ }^{4}$ Faculté de médecine, Sorbonne université, Paris, France. juliette.hadchouel@inserm.fr

chloe.rafael@gmail.com
> L'hypertension hyperkaliémique familiale (HHF) est une forme mendélienne rare d'hypertension artérielle associée à une hyperkaliémie et une acidose métabolique hyperchlorémique. Ces troubles sont corrigés par les diurétiques thiazidiques, inhibiteurs du cotransporteur $\mathrm{Na}^{+}$/ $\mathrm{Cl}^{-}\left(\mathrm{Na}^{+}-\mathrm{Cl}^{-}\right.$cotransporter, NCC) exprimé dans le tube contourné distal du néphron. Les premières analyses génétiques ont mis en évidence des mutations dans les gènes codant les sérine-thréonine kinases WNKl et WNK4 (with no (K) lysine) chez une minorité $(8,5 \%)$ de patients HHF [1]. De multiples études in vitro et in vivo ont permis de montrer que ces kinases stimulent l'activité du cotransporteur NCC grâce à une cascade de phosphorylations [2] $(\rightarrow)$.

$(\rightarrow)$ Voir la Synthèse de C. Rafael et al., En 2012, des nou$m / s n^{\circ} 3$, mars 2016, page 274

velles mutations dans les gènes CUL3 (cullin-3) et KLHL3 (kelch-like family member 3) ont été identifiées chez $47 \%$ des patients [3]. Les protéines CUL3 et KLHL3 font partie d'un complexe ubiquitine-ligase $\varepsilon 3$ permettant l'ubiquitination de protéines cibles en vue de leur dégradation par le protéasome. WNKl et WNK4 sont des substrats de ce complexe enzymatique [4] (Figure 1).

\section{Des mutations de gènes différents,} mais des conséquences communes Les mutations de WNKI sont de grandes délétions du premier intron du gène. Ces délétions entraînent une augmentation de l'expression de l'isoforme longue de la kinase WNKl (long WNKI, L-WNKl) dans le tube contourné distal du néphron [5]. Les mutations de WNK4 empêchent, quant à elles, l'interaction entre WNK4 et KLHL3, ce qui entraîne une diminution de l'ubiquitination et de la dégradation de la kinase par le protéasome [4]. Les mutations de $K L H L 3$ ont un effet équivalent car elles empêchent le recrutement des substrats ou la fixation de KLHL3 à CUL3. Dans les deux cas, ces mutations empêchent la dégradation des substrats du complexe KLHL3-CUL3, dont L-WNKI et WNK4, par le protéasome (Figure 1). Enfin, les mutations de CUL3 sont localisées dans des séquences impliquées dans l'épissage de l'exon 9, et entraînent l'absence de cet exon (par exon skipping) dans le transcrit mature [6]. Toutes les études in vitro ont montré que l'absence du domaine protéique codé par cet exon diminue l'activité du complexe ubiquitine-ligase, bien que les mécanismes ne soient pas encore définis avec précision [7]. Les mutations de CUL3 causent donc également une diminution de l'ubiquitination et de la dégradation de L-WNKl et WNK4. Par conséquent, toutes les mutations responsables de l'HHF ont un effet commun quel que soit le gène muté : une augmentation de l'abondance des protéines L-WNKl et/ ou WNK4.

Les patients porteurs d'une mutation de CUL3 souffrent d'une forme plus sévère de HHF

Comme nous l'avons mentionné, L-WNKl et WNK4 font partie d'une cascade de signalisation aboutissant à la phosphorylation et à l'activation du cotransporteur NCC. Les mutations de WNK1, WNK4, KLHL3 et CUL3 entraînent donc toutes une stimulation de l'activité de NCC. Le tableau clinique est d'ailleurs similaire chez tous les patients HHF quelle que soit la mutation dont ils sont porteurs. Toutefois, les patients porteurs de mutations de CUL3 ont une forme plus sévère de la maladie [6]. En effet, $94 \%$ d'entre eux souffrent d'hypertension artérielle avant l'âge de 18 ans, alors que ce taux n'est que de 10 à $17 \%$ chez les autres patients atteints d'HHF. Les valeurs de la kaliémie sont également beaucoup plus élevées chez ces patients (supérieures à $7 \mathrm{mM}$ en moyenne) (Tableau I) [6]. Enfin, la plupart d'entre eux présentent également un défaut de croissance ou de développement, plus rarement rapporté chez les autres patients. Les résultats d'études in vitro et in vivo indiquent que cette sévérité pourrait résulter d'une atteinte de la fonction vasculaire s'ajoutant à la rétention de $\mathrm{NaCl}$ causée par l'activation de NCC dans le tube contourné distal du néphron $[8,9]$.

\section{CUL3 inhibe la vasoconstriction en} stimulant la dégradation de RhoA Une première étude, de l'équipe dirigée par Curt Sigmund, a permis de montrer que la protéine RhoA (Ras homo$\log$ family member $A$ ) est une cible du complexe d'ubiquitination dépendant de CUL3. RhoA est une protéine $G$ monomérique de la famille des protéines Rho, 


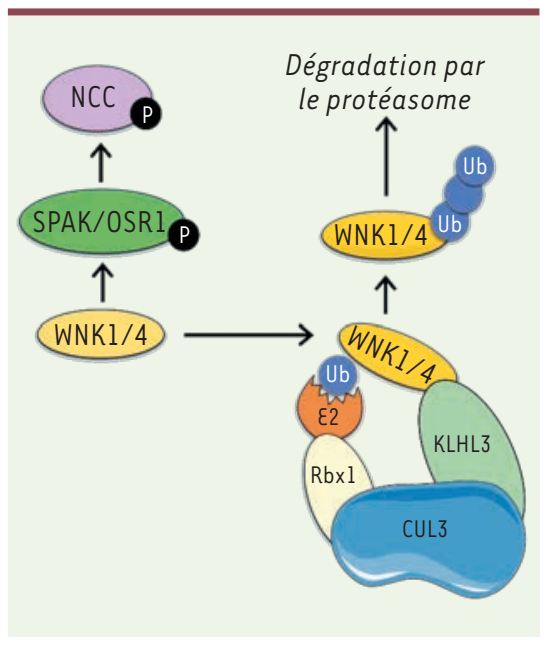

elle-même membre de la super-famille des protéines Ras [12] $(\rightarrow)$. Lorsque RhoA est stimulée dans les cellules

$(\rightarrow)$ Voir le Repères de H. Barelli et al., $\mathrm{m} / \mathrm{s} \mathrm{n}^{\circ} 4$, avril 2020, page 394

musculaires lisses vasculaires par des agents vasoconstricteurs comme l'angiotensine II, elle active la kinase ROCK (Rho-associated protein kinase), qui inhibe la phosphatase spécifique de la chaîne légère de la myosine (MLCP) et la déphosphorylation de la myosine, ce qui favorise la contraction vasculaire [10]. In vitro, l'inhibition de CUL3 ou la surexpression de la protéine CUL3 $\Delta 403-459$, délétée de la séquence codée par l'exon 9, entraîne une diminution de l'ubiquitination et de la dégradation de RhoA $[8,10]$.

CUL3 pourrait donc inhiber le tonus vasculaire en stimulant la dégradation de RhoA dans les cellules musculaires

Figure 1. Le complexe ubiquitine ligase $\varepsilon 3-C U L 3 / K L H L 3$ inhibe la voie de signalisation induite par WNKI et WNK4. Les kinases SPAK (Ste20-related proline alanine-rich kinase) et OSRI (oxidative stress response 1) sont phosphorylées et activées par les kinases WNKI et WNK4. Une fois activées, SPAK et OSRI activent NCC $\left(\mathrm{Na}^{+}-\mathrm{Cl}^{-}\right.$cotransporter) par phosphorylation. Le complexe ubiquitine ligase 83 CUL3/KLHL3 recrute WNK1 et WNK4 pour induire leur dégradation par le protéasome, ce qui entraîne une diminution de leur abondance. La diminution de l'abondance des kinases diminue l'activation de NCC, ce qui limite la réabsorption de $\mathrm{NaCl}$ dans le tube contourné distal du néphron (figure adaptée de [7]).

lisses vasculaires. Cette hypothèse a été confirmée in vivo par la même équipe. L'administration d'un inhibiteur des protéines CUL à des souris témoins provoque en effet une vasoconstriction et une hypertension artérielle. De même, les souris surexprimant la protéine CUL3 4403-459 spécifiquement dans les cellules musculaires lisses vasculaires ont une hypertension artérielle due à une stimulation de la vasoconstriction et à une inhibition de la vasorelaxation [9].

\section{Deux modèles murins pour comprendre} la sévérité de l'hypertension artérielle associée aux mutations CUL3

Ces études ont donc permis de montrer que l'inhibition de CUL3 et la surexpression de CUL3 4403-459 entraînent un dysfonctionnement vasculaire suite à la dérégulation de la voie de signalisation RhoA dans les cellules musculaires lisses des artères. Par ailleurs, l'analyse de souris porteuses d'une mutation de Cul3 semblable à celle des patients a confirmé que la délétion de la séquence codée par l'exon 9 dans la protéine CUL3 conduit effectivement à une diminution de la dégradation de WNK4, associée à une stimulation de NCC et à une hypertension artérielle [8]. Cependant, ces deux séries d'étude n'avaient pas permis de déterminer les contributions respectives des atteintes rénale et vasculaire dans la HHF. Afin d'étudier ces contributions, nous avons généré deux modèles de souris: les souris pgk-Cul3 $\Delta 9$, qui ont une délétion ubiquitaire de l'exon 9 de Cul3, et les souris sm22-Cul3 $\Delta 9$, porteuses de cette mutation uniquement dans les cellules musculaires lisses vasculaires (Figure 2) [11].

Les souris pgk-Cul3 $\Delta 9$ présentent tous les phénotypes associés à la HHF, c'està-dire une hyperkaliémie, une acidose métabolique hyperchlorémique et une hypertension artérielle sévère, associées à une activation de la voie de signalisation WNK4/NCC. Ces troubles tensionnels et métaboliques sont plus sévères que chez les souris $W n k 1^{+/ F H H t}$, porteuses d'une mutation intronique de Wnkl équivalente de celles des patients. Comme chez l'homme, les mutations de Cul3 chez la souris entraînent donc un phénotype plus sévère que les mutations de Wnkl. L'abondance et la phosphorylation de NCC sont en revanche similaires dans les deux

\begin{tabular}{ccccc}
\hline Gène muté & $\begin{array}{c}\text { Âge lors du dia- } \\
\text { gnostic (années) * }\end{array}$ & $\begin{array}{c}\text { Concentration } \\
\text { plasmatique } \\
\text { de } \mathbf{K}^{+}(\mathbf{m M}) * *\end{array}$ & $\begin{array}{c}\text { Concentration } \\
\text { plasmatique } \\
\mathbf{d}^{\prime} \mathbf{H C O 3}^{-}(\mathbf{m} \mathbf{M}) * *\end{array}$ & $\begin{array}{c}\text { Hypertensionartérielle } \\
\text { avant l'âge de 18 ans** }\end{array}$ \\
\hline CUL3 & $9 \pm 6$ & $7,5 \pm 0,9$ & $15,5 \pm 2,0$ & $94 \%$ \\
\hline KLHL3 & $24 \pm 18$ & $6,2 \pm 0,6$ & $17,2 \pm 2,5$ & $17 \%$ \\
\hline WNK4 & $28 \pm 18$ & $6,4 \pm 0,7$ & $20,8 \pm 2,3$ & $10 \%$ \\
\hline WNK1 & $36 \pm 20$ & $5,8 \pm 0,8$ & $22,4 \pm 4,6$ & $13 \%$ \\
\hline
\end{tabular}

Tableau I. Caractéristiques des patients atteints de HHF en fonction du type de mutation dont ils sont porteurs. Les valeurs indiquées correspondent à la moyenne \pm écart-type. Tests statistiques utilisés : ANOVA (pour l'âge lors du diagnostic et les concentrations plasmatiques de $\mathrm{K}^{+}$et d'HC03-) ou test exact de Fisher (pour l'hypertension artérielle); ${ }^{\star} p=0,0002 ;{ }^{* \star} p<0,0001$ (tableau adapté de [6]). 

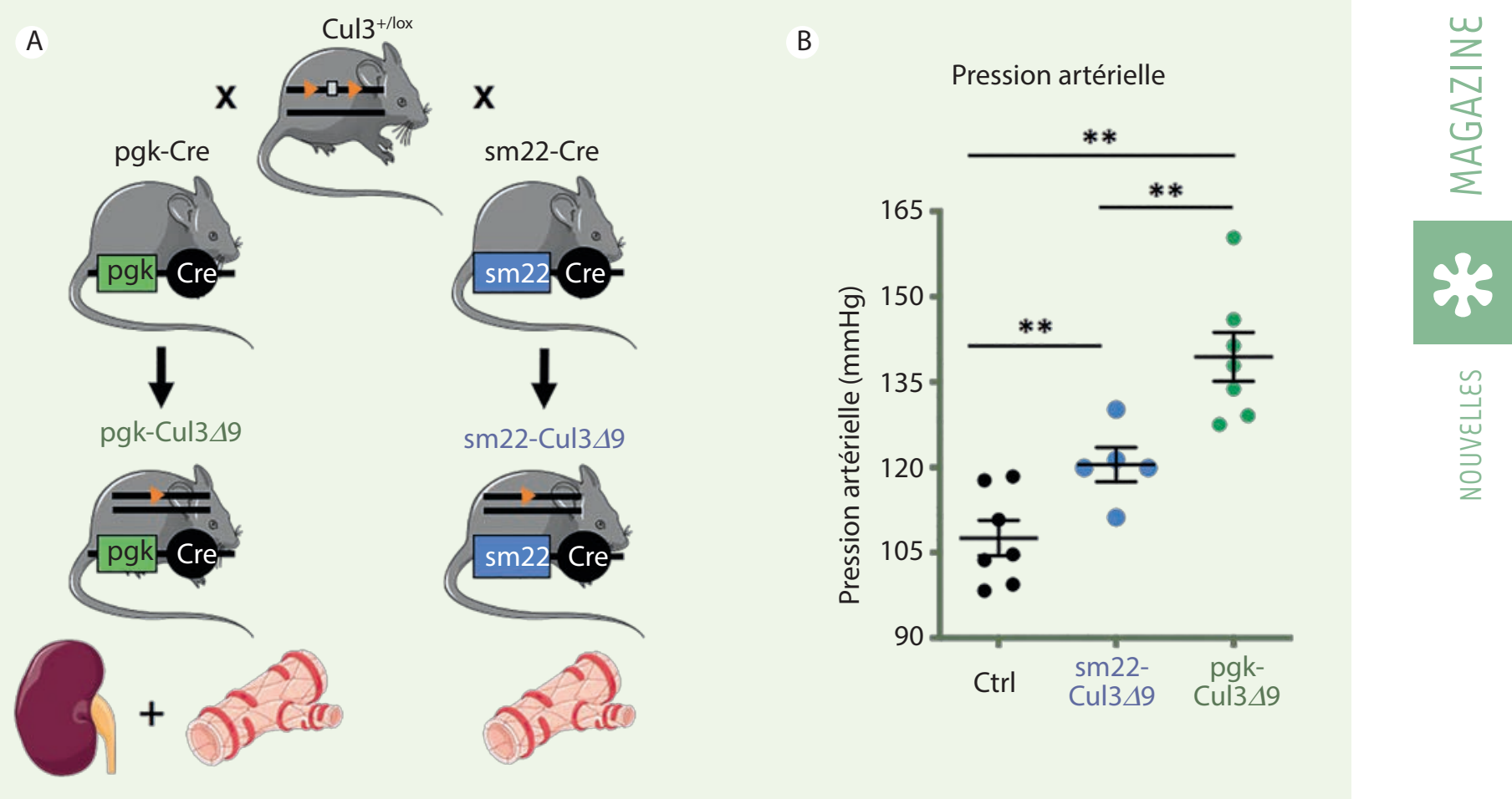

Figure 2. Les souris sm22-Cul3 $\Delta 9$ ont une hypertension artérielle moins sévère que les souris pgk-Cul3 $\Delta 9$. (A) Des femelles Cul3+/lox, porteuses de sites loxP de part et d'autre de l'exon 9 du gène Cul3, ont été croisées avec des mâles transgéniques pgk-Cre pour générer les animaux pgk-Cul3 $\Delta 9$, porteurs de la délétion hétérozygote ubiquitaire de l'exon 9 de Cul3. Les femelles Cul $3^{+/ l o x}$ ont également été croisées avec des mâles transgéniques sm22-Cre ${ }^{\mathrm{Tg}}$ pour générer les animaux sm22-Cul3 $\Delta 9$, porteurs de la délétion hétérozygote de l'exon 9 de Cul3 spécifiquement dans les cellules musculaires lisses vasculaires. (B) Les souris sm22-Cul3 $\Delta 9$ ont une hypertension artérielle modérée, tandis que les souris pgk-Cul3 $\Delta 9$ ont une hypertension artérielle sévère. La pression artérielle systolique a été mesurée par une méthode non-invasive de sphygmomanométrie («tail-cuff»)

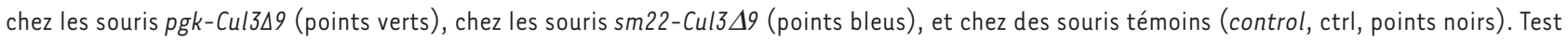
statistique : ANOVA à deux facteurs suivi de comparaisons multiples. ${ }^{* \star} p<0,0001$ (figure adaptée de [11]).

groupes de souris mutantes. Ce résultat indique que la sévérité des troubles métaboliques chez les souris pgk-Cul3 $\Delta 9$ n'est pas causée par une plus forte activation de NCC. Contrairement aux souris

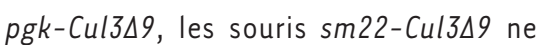
présentent aucun trouble métabolique, mais ont cependant une hypertension artérielle, bien qu'elle soit moins sévère que chez les souris pgk-Cul3 $\Delta 9$ (Figure 2). L'hypertension artérielle des souris sm22Cul349 résulte d'un défaut de la vasorelaxation consécutive à une surabondance de RhoA, comme dans le modèle de surexpression de CUL3 $4403-459$ restreinte aux cellules musculaires lisses vasculaires cité précédemment. Notre étude a donc permis de montrer pour la première fois que la sévérité de l'hypertension artérielle associée aux mutations de CUL3 chez les patients atteints d'HHF résulte des dysfonctionnements cumulés du néphron et du tonus vasculaire. Elle n'a cependant pas permis d'expliquer la sévérité des troubles métaboliques chez ces patients. La poursuite d'une recherche physiopathologique dans cette forme génétique de la maladie conduira peut-être à la découverte de nouvelles voies de régulation du transport ionique dans le rein, et plus particulièrement dans le tube contourné distal du néphron. $\diamond$

Severity of familial hyperkalemic hypertension caused by CUL-3 mutations: a story about kidneys and blood vessels

\section{LIENS D'INTÉRÊT}

Les auteures déclarent n'avoir aucun lien d'intérêt concernant les données publiées dans cet article.

\section{RÉFÉRENCES}

1. Wilson FH, Disse-Nicodème $S$, Choate KA, et al. Human hypertension caused by mutations in WNK kinases. Science 2001 ; 293 : 1107-12.

2. Rafael C, Chavez-Canales M, Hadchouel J. Réabsorption du sel et sécrétion du potassium par le néphron distal : vision nouvelle du rôle régulateur des kinases de la famille WNK. Med Sci (Paris) 2016 ; 32 : 274-80.

3. Louis-Dit-Picard H, Barc J, Trujillano D, et al. KLHL3 mutations cause familial hyperkalemic hypertension by impairing ion transport in the distal nephron. Nat Genet 2012 ; $44:$ 456-60.

4. Wakabayashi M, Mori T, Isobe K, et al. Impaired KLHL3-mediated ubiquitination of WNK4 causes human hypertension. Cell Rep 2013 ; 3 : 858-68.

5. Vidal-Petiot $\varepsilon$, Elvira-Matelot $\varepsilon$, Mutig K, et al. WNKIrelated familial hyperkalemic hypertension results from an increased expression of L-WNKI specifically in the distal nephron. Proc Natl Acad Sci USA 2013 ; 110 : 14366-71.

6. Boyden LM, Choi M, Choate KA, et al. Mutations in kelch-like 3 and cullin 3 cause hypertension and electrolyte abnormalities. Nature 2012 ; 482 : 98-102

7. Ferdaus MZ, McCormick JA. Mechanisms and controversies in mutant Cul3-mediated familial hyperkalemic hypertension. Am J Physiol Renal Physiol 2018 ; 314 : F915-20 


\section{RÉFÉRENCES}

8. Schumacher FR, Siew K, Zhang J, et al. Characterisation of the cullin- 3 mutation that causes a severe form of familial hypertension and hyperkalaemia. EMBO Mol Med 2015 ; 7 : 1285-306.

9. Agbor LN, Ibeawuchi SRC, Hu C, et al. Cullin-3 mutation causes arterial stiffness and hypertension through a vascular smooth muscle mechanism. $J \mathrm{Cl}$ Insight 2016 ; 1 : doi:10.1172/jci.insight.91015.

10. Pelham CJ, Ketsawatsomkron P, Groh S, et al. Cullin-3 regulates vascular smooth muscle function and arterial blood pressure via PPAR $\gamma$ and RhoA/Rhokinase. Cell Metab 2012 ; 16: 462-72.

11. Abdel Khalek W, Rafael C, Loisel-Ferreira I, et al. Severe arterial hypertension from cullin- 3 mutations

\section{NOUVELLE}

\section{Surexpression de PAX4 par thérapie génique}

\section{Un espoir pour le traitement du diabète de type 1}

Kevin Perge ${ }^{1,2}$, Assia Eljaafari ${ }^{2,4}$
> Dans un rapport de 2016, l'Organisation mondiale de la santé (OMS) reconnaît que le diabète est devenu un problème majeur de santé publique, du fait de la hausse spectaculaire du nombre de personnes diabétiques dans le monde durant les dernières décennies (estimé à 108 millions d'adultes en 1980, et 422 millions en 2014) associée à une morbidité et une mortalité très importantes [1]. Divers variants génétiques (mutations) du gène PAX4 (Paired Box gene 4), codant pour un facteur de transcription, ont été associés à la susceptibilité de développer un diabète de type 1 , un diabète de type 2 , ou un diabète monogénique de type MODY (maturity onset diabetes of the young) [2]. Du fait de son rôle essentiel dans la prolifération et la maturation des cellules pancréatiques sécrétrices d'insuline (cellules $\beta$ ) lors du développement [2], PAX4 apparaît comme une cible de choix pour une thérapie génique dans le diabète de type 1 (qui représente environ $10 \%$ de tous les cas de diabète), contre lequel il n'existe pas encore de traitement spécifique permettant de rétablir la fonction des cellules $\beta$, et qui a un impact considérable sur la qualité de vie [1]. Cette nouvelle étude offre un aperçu des trois mécanismes impliquant PAX4 dans les ilôts endocrines du pancréas (îlots de Langerhans) afin de proposer une éventuelle piste thérapeutique dans le traitement du diabète de type 1.

Rôle de PAX4 dans la prolifération et la survie des cellules $\boldsymbol{\beta}$ pancréatiques L'expression de PAX4 dans les cellules $\beta$ pancréatiques de l'adulte est faible comparativement à son expression embryonnaire. PAX4 joue cependant un rôle important dans le contrôle de la plasticité des cellules $\beta$ au cours de la vie, en partie lié à la prolifération et la survie de ces cellules [3]. En effet, la destruction sélective des cellules $\beta$ induite par la streptozotocine stimule l'expression du gène $P A X 4$, suggérant que dans des conditions de stress, PAX4 est induit en tant que mécanisme adaptatif de protection des cellules $\beta$ contre les agressions de l'environnement. L'effet protecteur de PAX4 sur les cellules $\beta$ passe par trois voies de signalisation [3-5]. La première est la voie $c-m y c / b c l-x l[3]$. Chez le rat, la surexpression constitutive de PAX4 dans les îlots de Langerhans au moyen d'un vecteur de type adénovirus recombinant stimule la prolifération et la survie des cellules $\beta$ grâce à la régulation concomitante de l'oncogène $c-m y c$ et $\mathrm{du}$ gène anti-apoptotique $b c l-x l$ [3]. L'oncogène $c-m y c$ est un important is caused by both renal and vascular effects. J Am Soc Nephrol $2019 ; 30: 811-23$

12. Barelli H, Camonis J, de Gunzburg J. Pierre Chardin, un pionnier de la découverte des gènes et protéines de la super famille Ras. Med Sci (Paris) 2020 ; $36: 394-8$

\author{
${ }^{1}$ Hospices civils de Lyon, Hôpital femme mère \\ enfant, Service d'endocrinologie pédiatrique, \\ 59 boulevard Pinel, 69677 Bron Cedex, France. \\ 2Université Claude Bernard Lyon 1, Lyon, \\ France. \\ ${ }^{3}$ Laboratoire CarMeN, Inserm U1060, \\ Université Claude Bernard Lyon 1, INRAE \\ U1397, INSA-Lyon, Lyon, France Clinical \\ Research Department, Hospices civils de \\ Lyon, Centre hospitalier Lyon-Sud, \\ Pierre-Bénite, France. \\ ${ }^{4}$ Do-it team, Hospices Civils de Lyon, Bâtiment \\ CENS-ELI, CHU Lyon Sud, Lyon, France. \\ kevin.perge@chu-lyon.fr
}

régulateur de la prolifération des cellules $\beta$, mais paradoxalement, il favorise simultanément leur apoptose [3]. Bcl-xl prévient l'apoptose induite par c-myc et favorise la prolifération des cellules $\beta$ en supprimant la voie mitochondriale de l'apoptose [3]. Il existe donc un couplage intime, régulé par PAX4, entre c-myc et $b c l-x l$ dans la promotion de la prolifération et de la survie des cellules $\beta$. Le deuxième mécanisme est l'inhibition de la voie NF-KB (nuclear factor-kappa B) [4]. La surexpression constitutive de PAX4 dans les cellules $\beta$ chez la souris, induit une réduction du taux de transcrits codant pour l'interleukine $1 \beta$ (IL$1 \beta$ ), la principale cytokine responsable de l'activation de la voie $N F-\kappa B$ et des transcrits de Nos2 (nitric oxide synthase 2 ), un gène cible de NF-KB [4]. Par ce mécanisme, PAX4 prévient les dommages exercés sur les cellules $\beta$ par l'hyperglycémie [4]. Enfin, PAX4 cible de nombreux gènes impliqués dans la protéostase, la translocation des protéines du réticulum endoplasmique vers l'appareil de Golgi, et I'homéostasie du calcium [5]. L'ensemble de ces régulations font de PAX4 un acteur essentiel de l'homéostasie du réticulum endoplasmique, d'autant plus importante en cas de stress, prévenant par ce biais la 